ARTICLE OPEN

\title{
Facile fabrication of paper-based flexible thermoelectric
}

\section{generator}

\author{
Zuoyuan Dong $\mathbb{D}^{1,3}$, Hua Liu ${ }^{2,3}$, Xin Yang ${ }^{1}$, Jichen Fan ${ }^{1}$, Hengchang $\mathrm{Bi}^{1}$, Chaolun Wang ${ }^{1}$, Yonghua Zhang ${ }^{1}$, Chen Luo $^{1}$, \\ Xinqian Chen $^{1}$ and Xing Wu iD $^{1 凶}$
}

Paper, as a foldable, pollution-free, and low-cost material, has become a suitable support substrate for producing flexible thermoelectric (TE) generators to realize waste heat recycling and the application of human-powered electronic devices. We propose a facile fabrication method to modify cellulose paper with inorganic TE powders via vacuum filtration, making a modified paper that possesses good thermoelectric properties. By connecting the modified paper to copper foils, flexible paper-based TE generators (PTGs) are fabricated. The obtained PTG with three units of P-N modules can generate an output voltage of $\sim 41.2 \mathrm{mV}$ at a temperature difference of $50 \mathrm{~K}$. Based on this modified paper, a thermal sensor that responds to heat sources, such as fingers, is proposed with a rapid response time of $0.25 \mathrm{~s}$. This work offers a promising strategy for the simple fabrication of PTGs, paving the way for achieving the commercial application of self-powered wearable electronics.

npj Flexible Electronics (2021)5:6; https://doi.org/10.1038/s41528-021-00103-1

\section{INTRODUCTION}

The invention of cellulose fiber-based paper has undoubtedly contributed greatly to the progress of society over thousands of years. Recently, paper has also become a popular and promising component used in flexible electronics due to its foldability, low cost, light weight, and environmental friendliness ${ }^{1-5}$. Some techniques, such as printing and magnetron sputtering, are perfectly combined with paper, making paper a substrate or skeleton to achieve creative applications in flexible field-effect transistors ${ }^{6}$, sensors ${ }^{7}$, and energy harvesting devices ${ }^{8}$. In particular, paper-based thermoelectric (TE) energy harvesting devices have been proposed to realize waste heat utilization on flexible surfaces $^{9-12}$. TE generators can directly convert heat into electrical energy from a temperature difference. Their efficiency is determined by the dimensionless figure of merit $Z T\left(Z T=S^{2} \sigma T / K\right)$, where $S$ is the Seebeck coefficient, $\sigma$ is the electrical conductivity, $T$ is the absolute temperature, and $K$ is the thermal conductivity. Current efforts tend to combine thermoelectricity and wearability to power portable electronic devices through temperature differences between the human body and environment ${ }^{13}$. The obstacle to commercialization of this concept is the difficulty in making lightweight and flexible inorganic TE materials. An effective way is to make TE materials into films or papers ${ }^{14,15}$. In recent years, some promising TE materials, such as $\mathrm{Bi}_{2} \mathrm{Te}_{3}$ and $\mathrm{Sb}_{2} \mathrm{Te}_{3}$, have been prepared for paper-based TE generators (PTGs) by combining with paper, thereby realizing light and flexible TE generators. Rojas et al. ${ }^{9}$ prepared a flexible, paper-based origami TE generator by magnetron sputtering, and its optimized output power reached $\sim 80 \mathrm{nW}$ at a temperature difference of $75 \mathrm{~K}$. Zhao et al. ${ }^{10}$ fabricated a transparent and flexible PTG using modified distributor printing technology to obtain a maximum voltage of $\sim 8.3 \mathrm{mV}$ at a temperature difference of $35 \mathrm{~K}$. These works successfully circumvent the brittleness of inorganic semiconductors to achieve flexible PTGs and demonstrate their possible application.
There are some issues, such as complicated preparation processes, high preparation costs, and poor performance in current PTGs. Therefore, it is necessary to develop more efficient and inexpensive preparation techniques to achieve the large-scale application of PTGs. In this work, we explore a simple method to modify cellulose paper and use modified paper to fabricate PTGs. In this method, cellulose paper is modified by infiltrating inorganic TE materials (doped $\mathrm{Bi}_{2} \mathrm{Te}_{3}$ and $\mathrm{Sb}_{2} \mathrm{Te}_{3}$ ) into a paper matrix via a vacuum-assisted filtration process. The foldable PTGs are fabricated by connecting the cut modified paper to copper foils. The obtained PTG with three units of $\mathrm{P}-\mathrm{N}$ modules can generate $\sim 41.2 \mathrm{mV}$ at a temperature difference of $50 \mathrm{~K}$. Furthermore, based on the modified paper, a finger touch sensor is fabricated, which can respond quickly to heat sources with a relaxation time of $0.25 \mathrm{~s}$. This work demonstrates the feasibility of PTGs and thermal sensors prepared by modified cellulose paper.

\section{RESULTS AND DISCUSSION}

\section{Fabrication of the generators}

Figure 1a shows the fabrication process of modified cellulose papers and PTGs. The N- and P-type modified papers are prepared through the vacuum filtration method. The $\mathrm{N}$-type material is $\mathrm{Bi}_{2} \mathrm{Te}_{3}$ doped with $\mathrm{Se}$, and the P-type material is $\mathrm{Sb}_{2} \mathrm{Te}_{3}$ doped with $\mathrm{Bi}$. First, N-type TE powder $(100 \mathrm{mg})$ and sodium carboxymethyl cellulose (CMC-Na) are dispersed in $30 \mathrm{ml}$ deionized water at a mass ratio of 12 to 1 , and the mixture is stirred for $10 \mathrm{~min}$ to form an N-type TE dispersion. CMC-Na is used to enhance the stability of the TE dispersion. The dispersion is then sonicated for 40 min to reduce the particle size of the TE powder and to allow it to fully disperse. The treated dispersion is poured into a vacuum flask for suction filtration. A pump is connected to the suction bottle mouth to maintain a pressure difference. The relative pressure is $-0.06 \mathrm{MPa}$, and suction filtration is conducted for 4-5 h. The P-type TE powder is prepared with the same procedure. The experimental process of vacuum-assisted filtration has been

\footnotetext{
'Shanghai Key Laboratory of Multidimensional Information Processing, School of Communication and Electronic Engineering, East China Normal University, Shanghai, China. ${ }^{2}$ Department of Cardiology, Shanghai Geriatric Medical Center, Shanghai, China. ${ }^{3}$ These authors contributed equally: Zuoyuan Dong, Hua Liu. ${ }^{凶}$ email: xwu@ee.ecnu.edu.cn
} 

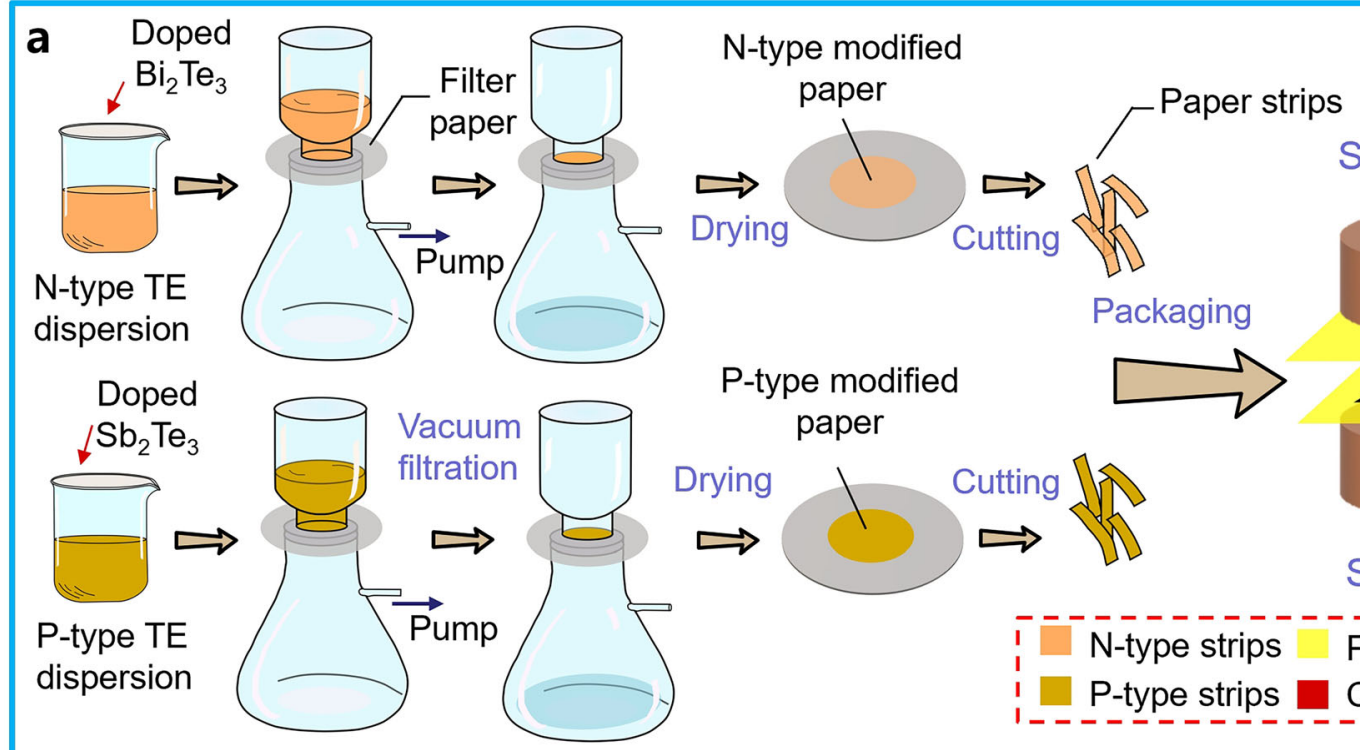

\section{P-type modified} paper

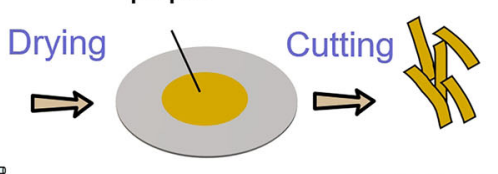

\section{Stress}

P-type TE dispersion
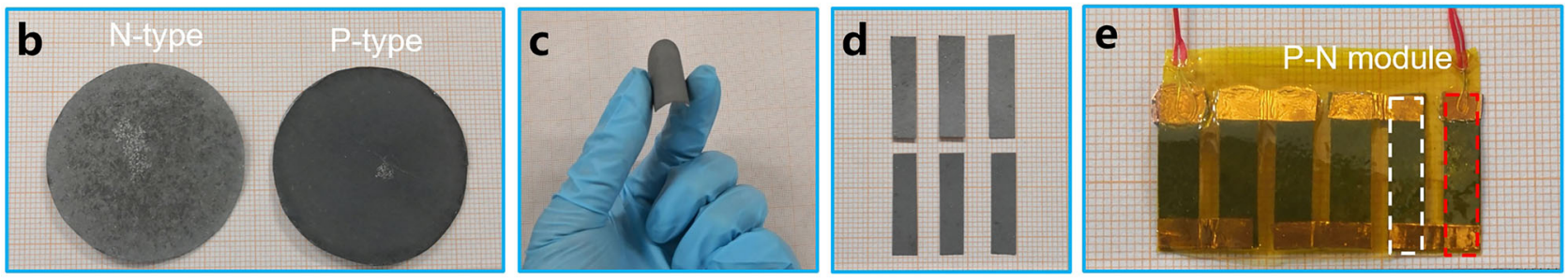

Fig. 1 Preparation process of flexible paper-based thermoelectric generator (PTG) and the photos display. a Schematic diagram of the fabrication process for PTGs. The N- and P-type modified papers are prepared through the vacuum filtration method, cut into small strips and connected with copper foil in an alternating sequence. b-e Photographs of the complete modified papers obtained by the vacuum filtration method (b), displaying their high flexibility (c), the small $5 \mathrm{~mm} \times 20 \mathrm{~mm}$ strips (d), and the PTG with three units of P-N modules (e). The smallest grid point of the background grid paper represents $1 \mathrm{~mm}$.

described in our reported works ${ }^{16,17}$. Relying on the pressure difference, the TE particles infiltrate into and adhere to the filter paper, and the modified papers shown in Fig. $1 \mathrm{~b}$ are formed after drying. Changing the quality of TE powder results in modified paper with different weight percentages (the mass of TE particles as a percentage of the total mass of modified paper). In this work, we prepare modified papers in three different percentages: $37 \%$ (modified paper $_{37}$ ), 59\% (modified paper ${ }_{59}$ ), and 70\% (modified paper $_{70}$ ), with 100,200 , and $300 \mathrm{mg}$ of TE powders, respectively. The modified papers display excellent flexibility, which is shown in Fig. 1c. Subsequently, the $\mathrm{N}$ - and P-type modified papers are cut into identical strips with dimensions of $5 \mathrm{~mm} \times 20 \mathrm{~mm}$, which are shown in Fig. 1d. After this, the small strips are used as TE legs and are alternately connected to copper foils in the form of an "N-P-N-P" sequence to form a conductive path. Both sides of the obtained series of TE legs are covered with polyimide (PI) for packaging and then flattened with a pressure anvil at $10 \mathrm{MPa}$ to enhance the compactness of the TE particles. After the wires are attached on the ends of the TE legs, the flexible PTG shown in Fig. $1 \mathrm{e}$ is finally fabricated, which is comprised of several pairs of $\mathrm{P}-\mathrm{N}$ modules. This is a facile and inexpensive method for preparing flexible TE generators and can be applied on a large scale.

\section{General characterization}

The cross-sectional morphology of the modified paper was characterized by scanning electron microscopy (SEM). For comparison, Fig. 2a, b shows the cross-section SEM images of the pristine paper at scale bars of 100 and $10 \mu \mathrm{m}$. The inset of Fig. 2a is a photograph of filter paper used in the experiment. Figure $2 \mathrm{c}$ provides the cross-section micromodel, which shows that paper is composed of adjacent and interlaced cellulose fibers. Figure $2 \mathrm{~d}$, e shows the SEM images of the N-type modified paper at the corresponding scale. In Fig. 2d, the upper layer of the modified paper is enlarged in the insert with a scale bar of $50 \mu \mathrm{m}$, and the lower layer is enlarged in Fig. 2e. Through vacuum filtration, some of the particles are deposited on the surface of the paper, which forms a compact layer called the deposition layer, while some of the particles penetrate into the paper and adhere to the cellulose fibers, forming an infiltration layer. The final modified paper consists of a deposition layer and infiltration layer, and its structural model is illustrated in Fig. 2f. Due to the same treatment procedure, the sizes of the $\mathrm{N}$ - and P-type powders are similar (less than $10 \mu \mathrm{m})$. The TE grains in the deposition layer are compact and are adjacent to each other to form a conductive path. The grains that infiltrate into the paper are used as a conductive supplement, and the cellulose fiber ensures the flexibility of the modified paper.

The elemental composition of the active materials in the modified paper was characterized by transmission electron microscopy (TEM). The P-type TE material is $\mathrm{Sb}_{2} \mathrm{Te}_{3}$ doped with $\mathrm{Bi}$, and the $\mathrm{N}$-type is $\mathrm{Bi}_{2} \mathrm{Te}_{3}$ doped with $\mathrm{Se}$. Their crystalline structures are exhibited in Fig. $2 \mathrm{~g}$, $\mathrm{h}$, which show that $\mathrm{Sb}_{2} \mathrm{Te}_{3}$ and $\mathrm{Bi}_{2} \mathrm{Te}_{3}$ are hexagonal crystalline structures formed by stacking quintuple layers. The two quintuple layers are connected by weak van der Waals forces. Figure $2 \mathrm{i}$, j are high-resolution TEM (HRTEM) images of $\mathrm{Sb}_{2} \mathrm{Te}_{3}$ doped with $\mathrm{Bi}$ and $\mathrm{Bi}_{2} \mathrm{Te}_{3}$ doped with $\mathrm{Se}$, respectively. The corresponding fast Fourier transform (FFT) images indicate the hexagonal structure of both $\mathrm{Sb}_{2} \mathrm{Te}_{3}$ and $\mathrm{Bi}_{2} \mathrm{Te}_{3}$. Both the HRTEM and FFT images show the good crystallinity of $\mathrm{Sb}_{2} \mathrm{Te}_{3}$ and $\mathrm{Bi}_{2} \mathrm{Te}_{3}$. Figure $2 \mathrm{k}, \mathrm{I}$ are the energy 


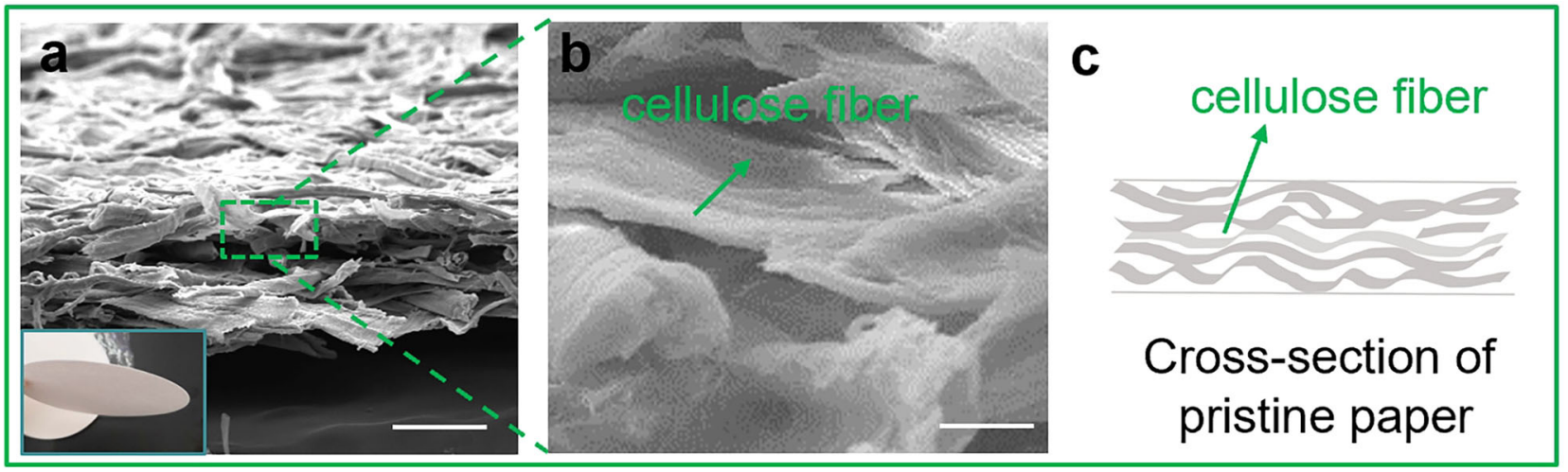

V. Vacuum filtration
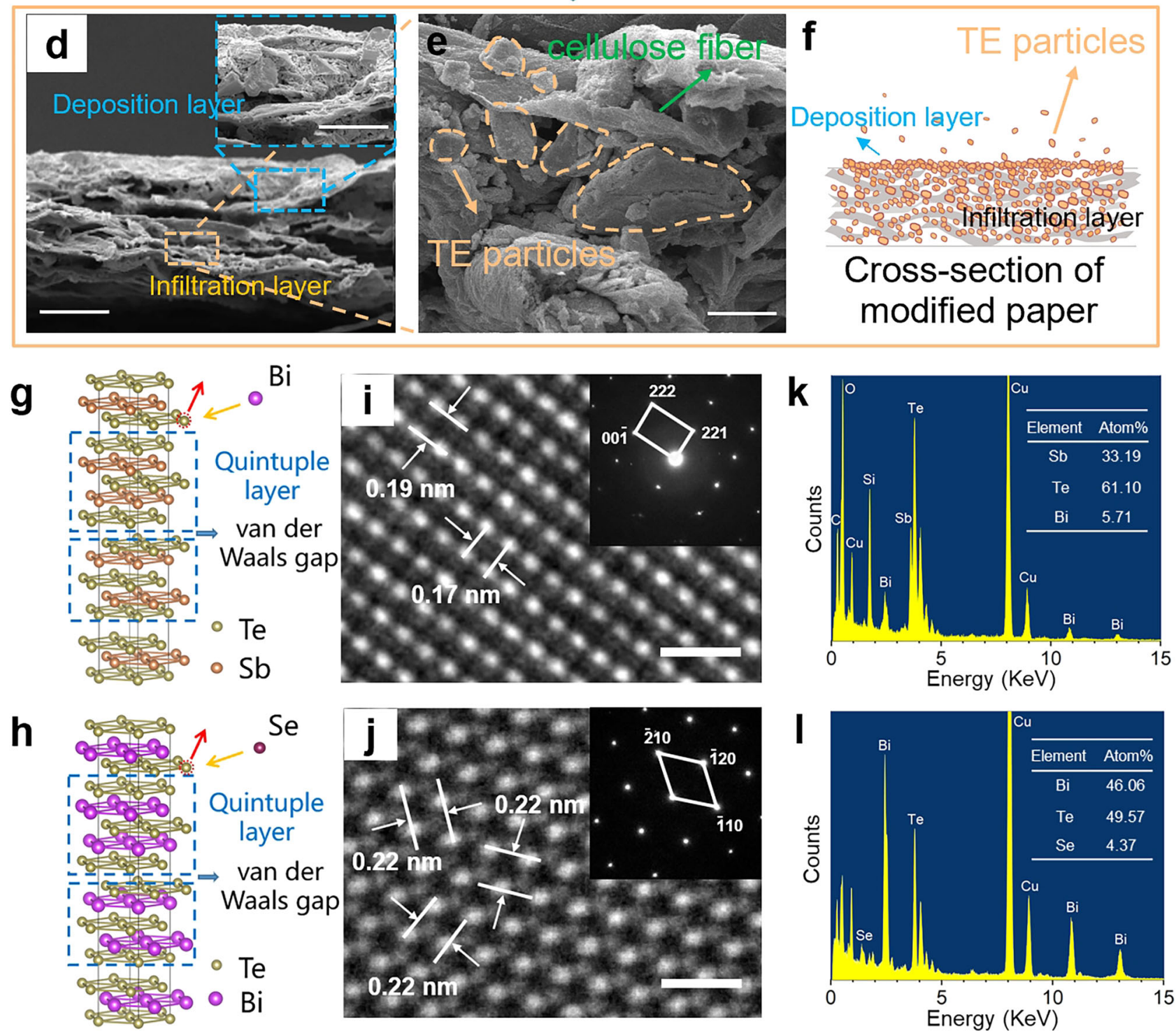

Fig. 2 Microscopic characterizations of pristine and modified papers with SEM and particles with high-resolution TEM. a Cross-sectional SEM images of the pristine paper with scale bars of $100 \mu \mathrm{m}$ and $\mathbf{b} 10 \mu \mathrm{m}$. The inset of $\mathbf{a}$ is a photograph of the filter paper used in the experiment. c Simple structural model of the filter paper with the adjacent cellulose fiber. $\mathbf{d}$ Cross-sectional SEM image of the N-type modified paper with a scale bar of $100 \mu \mathrm{m}$. The insert is an enlarged view of the deposition layer on the upper part of the modified paper, and the scale bar is $50 \mu \mathrm{m}$. e Enlarged view of the infiltration layer marked with an orange rectangle in (d). The TE particles are less than $10 \mu \mathrm{m}$. The SEM images are from the $\mathrm{N}$-type modified paper. $\mathbf{f}$ Micromodel of the modified paper composed of a deposition layer and infiltration layer. $\mathbf{g}$ Crystalline structures of $\mathrm{Sb}_{2} \mathrm{Te}_{3}$ doped with $\mathrm{Bi}$ and $\mathbf{h} \mathrm{Bi}_{2} \mathrm{Te}_{3}$ doped with $\mathrm{Se}$. i High-resolution TEM images and the corresponding fast Fourier transform (FFT) images of $\mathrm{Sb}_{2} \mathrm{Te}_{3}$ doped with $\mathrm{Bi}$ and $\mathbf{j} \mathrm{Bi}_{2} \mathrm{Te}_{3}$ doped with Se. The scale bars are $0.5 \mathrm{~nm}$. $\mathbf{k}$ Energy dispersive spectroscopy (EDS) results of $\mathrm{Sb}_{2} \mathrm{Te}_{3}$ doped with $\mathrm{Bi}$ and $\mathrm{I} \mathrm{Bi}_{2} \mathrm{Te}_{3}$ doped with Se. The atomic percentages of the corresponding elements are listed in the inserted table. 
a

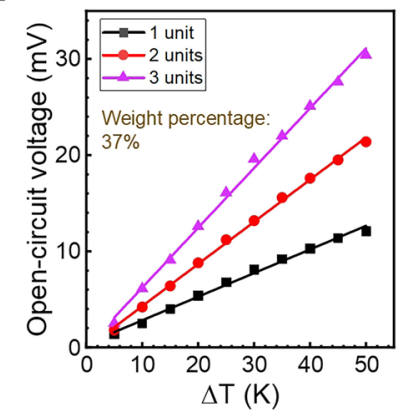

e

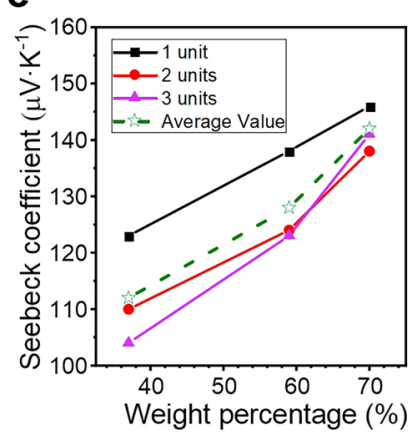

b

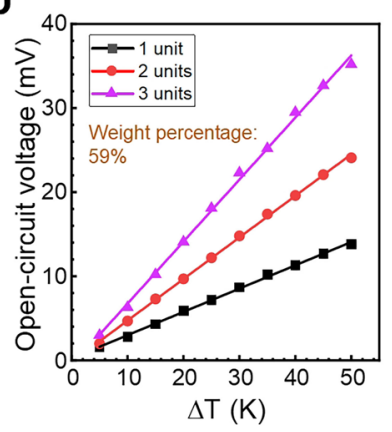

f

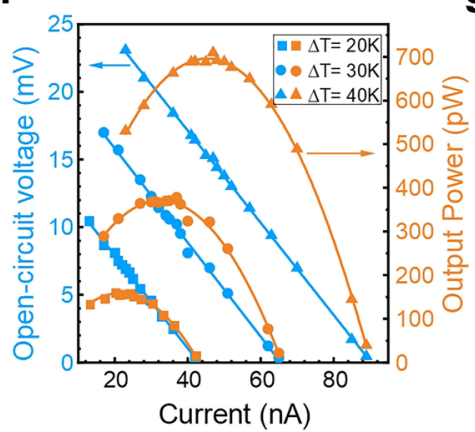

C

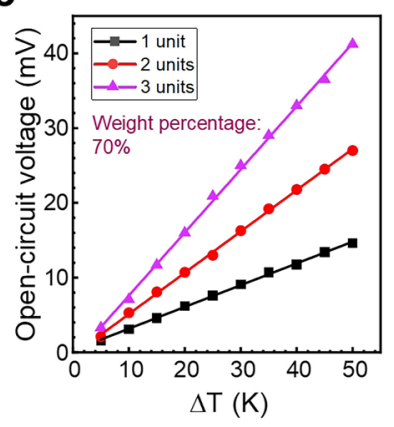

g

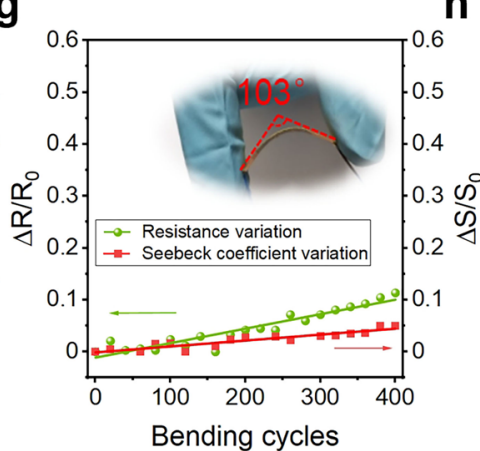

d

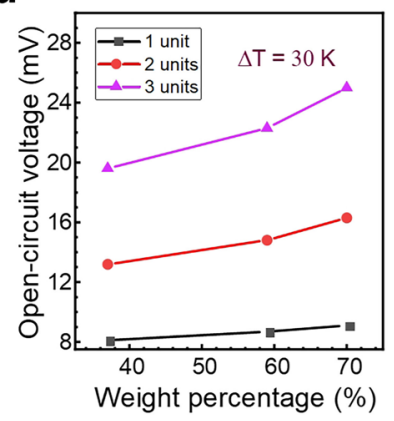

h

Fig. 3 TE performance tests of the PTGs. a-c Open-circuit voltage of the PTGs prepared by modified paper 37 (a), modified paper 59 (b), and modified paper $_{70}$ (c) at temperature differences of 5 to $50 \mathrm{~K}$. d Open-circuit voltage of the PTGs versus the three weight percentages at a temperature difference of $30 \mathrm{~K}$. e Seebeck coefficients (absolute value) of a single leg of the PTGs at the three weight percentages. $\mathbf{f}$ Output voltage and output power curves according to the various external circuit currents. $g$ Changes in the resistance and Seebeck coefficient after 400 bending cycles. The insert is the schematic diagram of the bending test. $\mathbf{h}$ Photograph of human skin generating electricity with the PTGs.

Table 1. Comparison of the reported Seebeck coefficient of a single leg of the flexible TE devices at room temperature.

\begin{tabular}{llll}
\hline TE materials & Method & Seebeck coefficient $\left(\mu \mathrm{V} \cdot \mathrm{K}^{-1}\right)$ & Reference \\
\hline $\mathrm{Bi}_{2} \mathrm{Te}_{3} /$ paper composite & Modified distributor printing technology & 60 & 10 \\
$\mathrm{PEDOT}: \mathrm{PSS}$ & Screen-printing & 99 & 18 \\
$\mathrm{PEDOT}$ nanowires & Vacuum-assisted filtration & 25.5 & 19 \\
$\mathrm{Bi}_{2} \mathrm{Te}_{3}$ and $\mathrm{Sb}_{2} \mathrm{Te}_{3}$ modified paper & Vacuum-assisted filtration & 142 & This work \\
\hline
\end{tabular}

dispersive spectroscopy (EDS) results of $\mathrm{Sb}_{2} \mathrm{Te}_{3}$ and $\mathrm{Bi}_{2} \mathrm{Te}_{3}$, respectively. The atomic percentages indicate that $\mathrm{Sb}: \mathrm{Te}: \mathrm{Bi}$ is approximately 33:61:6 and Bi:Te:Se is approximately 46:50:4. The specific composition of the $\mathrm{N}$ - and P-type active materials has been clarified.

\section{Performance of the generators}

PTGs with 1-3 units of P-N modules are fabricated and packaged with PI. The TE performance of the PTGs was tested, as shown in Fig. 3a-c shows graphs of the open-circuit voltage of PTGs with different weight percentages of active materials versus the temperature difference. At the same weight percentage, the open-circuit voltage increases proportionally with the units of the $\mathrm{P}-\mathrm{N}$ modules. The largest voltage reaches $\sim 41.2 \mathrm{mV}$ at a $\Delta T$ of $50 \mathrm{~K}$ and a weight percentage of $70 \%$. The open-circuit voltages with different weight percentages at a $\Delta T$ of $30 \mathrm{~K}$ are summarized in Fig. 3d. This figure shows that as the weight percentage increases, the generated voltage increases because more active materials participate in power generation. In regard to the weight percentages at $37 \%, 59 \%$, and $70 \%$, the PTGs with three units of $\mathrm{P}-\mathrm{N}$ modules generate open-circuit voltages of $\sim 19.6 \mathrm{mV}$, $\sim 22.3 \mathrm{mV}$ and $\sim 25 \mathrm{mV}$ at a $\Delta T$ of $30 \mathrm{~K}$, respectively. The Seebeck coefficient is an important parameter to characterize TE performance, which is defined as the magnitude of the generated TE voltage per degree of temperature difference. The definition can be expressed as

$S=\frac{d V}{d T}$

where $S$ is the Seebeck coefficient and $V$ is the TE voltage. Thus, the Seebeck coefficients of the flexible PTGs are obtained by calculating the single leg of PTGs, which are shown in Fig. 3e. For

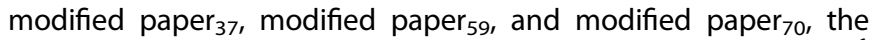
average Seebeck coefficients are 112,128 , and $142 \mu \mathrm{V} \mathrm{K}^{-1}$ (absolute value), respectively. Compared to previous reports on flexible TE generators, which are listed in Table $1^{10,18,19}$, the above values are impressive. Of course, the high efficiency of TE generators is not determined by a single parameter, which requires us to make further efforts to this end. Figure $3 f$ shows the variations in the external circuit voltage and output power with current in the PTG with three units of modules. As the load resistance increases, the current in the circuit decreases, and the voltage across the resistor increases. The value of the output power is the product of the external circuit voltage and current. 

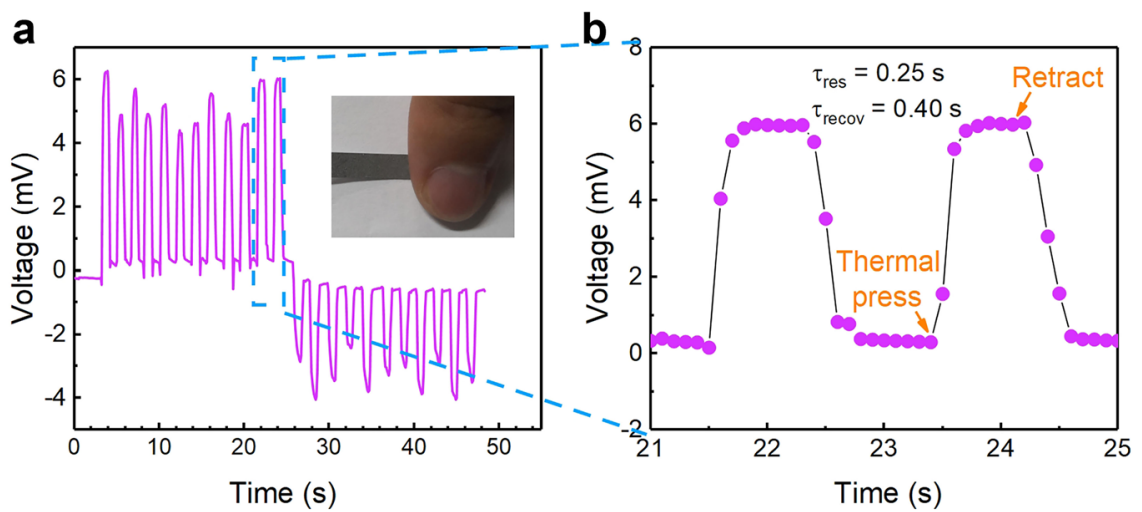

Fig. 4 Sensor response of the modified paper with a finger touch. a Voltage response with a finger touch at a frequency of approximately $1 \mathrm{~s}^{-1}$. The insert is the illustration of the finger touch test. $\mathbf{b}$ Enlarged view of the blue dashed box in (a).

The current can be obtained by

$I=\frac{U_{o}}{R_{i}+R_{L}}$

where $U_{o}$ is the open-circuit voltage of the TE device, $R_{i}$ is the internal resistance of the PTG, and $R_{L}$ is the load resistance of the circuit. The external circuit voltage $U_{e}$ can be expressed as

$U_{e}=I \cdot R_{L}$

and the output power $P$ can be expressed as

$P=U_{e} \cdot I$

In Fig. 3f, it can be observed that the maximum output power is $\sim 709 \mathrm{pW}$ at a $\Delta T$ of $40 \mathrm{~K}, \sim 351 \mathrm{pW}$ at a $\Delta T$ of $30 \mathrm{~K}$, and $\sim 155 \mathrm{pW}$ at a $\Delta T$ of $20 \mathrm{~K}$. The biggest reason for the output power being at a low level is that the internal resistance of the device is too large, which is over $50 \mathrm{k} \Omega$ for a single leg in the PTG. The durability of PTGs is tested with repeated bending cycles. Figure $3 \mathrm{~g}$ shows that the variation in internal resistance is less than $10 \%$ after 400 bending cycles with a bending angle of approximately $103^{\circ}$, and the Seebeck coefficient is less than $5 \%$. The good stability and durability indicate the potential application of the modified paper in flexible heat source identification devices. Flexible PTGs can be applied to human skin for power generation using the temperature difference between the ambient air and body. Figure $3 \mathrm{~h}$ displays the PTG attached to the wrist of a human body, which produces an open-circuit voltage of $2.38 \mathrm{mV}$ at a temperature difference of $5-6 \mathrm{~K}$. This observation demonstrates that these PTGs have potential applications for human energy harvesting.

Flexible modified paper can also be used as a thermal sensor to achieve a rapid response to contacting a heat source. Figure $4 a$ shows the voltage response of the flexible modified paper with a finger touch, and Fig. $4 \mathrm{~b}$ is the enlarged view in the blue dashed box of Fig. 4a. The inset shows the test process of the sensor response. When the finger is completely pressed on one side of the paper for heating, the open-circuit voltage of the paper reaches approximately $5 \mathrm{mV}$. After $25 \mathrm{~s}$, the position touched by the finger changes from one terminal of the modified paper to the other, and the polarity of the voltage alternates accordingly. The graphs depict that the voltage increases and decreases sharply with the finger touch at a frequency of approximately $1 \mathrm{~s}^{-1}$, illustrating that the modified paper is sensitive to changes in external temperature. It is noted that the maximum voltage for each cycle is not consistent, which is due to the different temperature changes induced by the finger touch. From Fig. $4 \mathrm{~b}$, a response time $\left(\tau_{\text {res }}\right)$ of $0.25 \mathrm{~s}$ and a recovery time $\left(\tau_{\text {recov }}\right)$ of $0.40 \mathrm{~s}$ are obtained. The response time is defined as the time required for the sensor to move from the baseline to $90 \%$ of its maximum voltage after finger pressing, and the recovery time is the time to move from $90 \%$ of its maximum voltage back to the baseline value after retracting the finger. Paper-based thermal sensors are expected to enable the self-powered control of wearable electronics.

In summary, $\mathrm{N}$ - and P-type modified paper and flexible PTGs were simply fabricated via vacuum filtration, and a high output voltage was obtained. Regarding the modified paper, some of the particles were deposited on the surface of the paper to form a deposition layer, and some of the particles penetrated into the paper to form an infiltration layer. The modified paper comprised of these two layers formed a conductive path. The TE particles were characterized by HRTEM and EDS, which showed that the specific component of the P-type TE material was SbTeBi with a ratio of approximately 33:61:6 and that the N-type TE material was BiTeSe with a ratio of approximately 46:50:4. The modified paper possessed a high Seebeck coefficient of $142 \mu \mathrm{V} \mathrm{K}^{-1}$, and PTGs with three units of $\mathrm{P}-\mathrm{N}$ modules could obtain a maximum output power of $\sim 709 \mathrm{pW}$ at a $\Delta T$ of $40 \mathrm{~K}$. Furthermore, based on the TE effect, a paper-based thermal sensor with a response time of $0.25 \mathrm{~s}$ was investigated. This work demonstrates the feasibility of PTGs and paper-based thermal sensors prepared by vacuum-assisted filtration.

\section{METHODS}

\section{Materials}

Doped $\mathrm{Bi}_{2} \mathrm{Te}_{3}$ (powder, 99.99\%) and $\mathrm{Sb}_{2} \mathrm{Te}_{3}$ (powder, 99.99\%) were purchased from Qijin New Material (Quanzhou, China). CMC-Na was purchased from Shandong Usolf Chemical Technology Company (Linyi, China). Medium-speed qualitative filter paper with a diameter of $9 \mathrm{~cm}$ was used in the experiment.

\section{Characterization and testing}

The SEM images were obtained by using an FEI XL30 FEG with an accelerating voltage of $20 \mathrm{kV}$. To conduct electricity, the cross-sections of the paper and TE film were covered with $20 \mathrm{~nm}$ of Au. To obtain an intact cross-section, the paper and paper-based TE film were broken after cooling with liquid nitrogen. The structural and compositional characterization of the samples were carried out by a JEM-ARM300F equipped with EDS at an accelerating voltage of $300 \mathrm{kV}$. TEM samples were prepared by the ultrasonic processing and centrifugation of the powder samples. Then, the supernatants of the centrifuged samples were drop cast on lacey carbon films and dried naturally for TEM characterization.

A heating stage (NWJ-3020) was used to heat the PTGs, and a thermocouple was used to monitor the real-time temperature. The voltage and sensor tests were carried out on an electrochemical workstation (CHI660E), and the current was measured by a picoammeter (Keithley Model 6485).

\section{DATA AVAILABILITY}

The datasets generated and analyzed during the current study are available from the corresponding authors upon reasonable request. 
Received: 25 March 2020; Accepted: 4 February 2021; Published online: 17 March 2021

\section{REFERENCES}

1. Cinti, S., Moscone, D. \& Arduini, F. Preparation of paper-based devices for reagentless electrochemical (bio)sensor strips. Nat. Protoc. 14, 2437-2451 (2019).

2. Lien, D.-H. et al. All-printed paper memory. ACS Nano 8, 7613-7619 (2014).

3. Hyun, W. J., Park, O. O. \& Chin, B. D. Foldable graphene electronic circuits based on paper substrates. Adv. Mater. 25, 4729-4734 (2013).

4. Lin, Y., Gritsenko, D., Liu, Q., Lu, X. \& Xu, J. Recent advancements in functionalized paper-based electronics. ACS Appl. Mater. Interfaces 8, 20501-20515 (2016).

5. Ha, D., Fang, Z. \& Zhitenev, N. B. Paper in electronic and optoelectronic devices. Adv. Electron. Mater. 4, 1700593 (2018).

6. Fortunato, E. et al. High-performance flexible hybrid field-effect transistors based on cellulose fiber paper. IEEE Electron Device Lett. 29, 988-990 (2008).

7. Beduk, T. et al. A paper-based inkjet-printed PEDOT:PSS/ZnO sol-gel hydrazine sensor. Sens. Actuators B 306, 127539 (2020).

8. Zhang, H. et al. A novel rhombic-shaped paper-based triboelectric nanogenerator for harvesting energy from environmental vibration. Sens. Actuators A 302, 111806 (2020).

9. Rojas, J. P. et al. Paper-based origami flexible and foldable thermoelectric nanogenerator. Nano Energy 31, 296-301 (2017).

10. Zhao, X. et al. Fabrication of transparent paper-based flexible thermoelectric generator for wearable energy harvester using modified distributor printing technology. ACS Appl. Mater. Interfaces 11, 10301-10309 (2019).

11. Ferhat, $\mathrm{S}$. et al. Flexible thermoelectric device based on TiS2(HA)x n-type nanocomposite printed on paper. Org. Electron. 68, 256-263 (2019).

12. Zhao, X. et al. A honeycomb-like paper-based thermoelectric generator based on $\mathrm{a} \mathrm{Bi}_{2} \mathrm{Te}_{3}$ /bacterial cellulose nanofiber coating. Nanoscale 11, 17725-17735 (2019).

13. Qu, D., Huang, X., Li, X., Wang, H. \& Chen, G. Annular flexible thermoelectric devices with integrated-module architecture. npj Flex. Electron. 4, 1 (2020).

14. Choi, J., Lee, J. Y., Lee, S.-S., Park, C. R. \& Kim, H. High-performance thermoelectric paper based on double carrier-filtering processes at nanowire heterojunctions. Adv. Energy Mater. 6, 1502181 (2016).

15. Lu, Y. et al. Good performance and flexible PEDOT:PSS $/ \mathrm{Cu}_{2} \mathrm{Se}$ nanowire thermoelectric composite films. ACS Appl. Mater. Interfaces 11, 12819-12829 (2019).

16. Liu, S. et al. Ultrafast dynamic pressure sensors based on graphene hybrid structure. ACS Appl. Mater. Interfaces 9, 24148-24154 (2017).

17. Tian, $X$. et al. Dual-mode sensor and actuator to learn human-hand tracking and grasping. IEEE Trans. Electron Devices 66, 5407-5410 (2019).

18. Zheng, C. et al. A flexible self-powered sensing element with integrated organic thermoelectric generator. Adv. Mater. Technol. 4, 1900247 (2019).

19. Ni, D., Song, H., Chen, Y. \& Cai, K. Free-standing highly conducting PEDOT films for flexible thermoelectric generator. Energy 170, 53-61 (2019).

\section{ACKNOWLEDGEMENTS}

This work is supported by NSFC under grant No. 62074057, Projects of Science and Technology Commission of Shanghai Municipality Grant nos. (19ZR1473800 and 18DZ2270800), Young Elite Scientists Sponsorship Program by CAST (YESS), and the Fundamental Research Funds for the Central Universities. The authors thank the help of Xiao-Jia Chen.

\section{AUTHOR CONTRIBUTIONS}

X.W., Z.D., and H.L. conceived and designed the experiments. Z.D., J.F., and C.W. fabricated and measured the PTGs. H.L. C.L., X.C., and X.Y. characterized and analyzed the materials. X.W., H.B., C.W., and Y.Z. supervised the overall research. Z.D., H.L. H.B., X.W., and C.L. contributed to the writing of the paper. Z.D. and H.L. are contributed equally to the paper.

\section{COMPETING INTERESTS}

The authors declare no competing interests.

\section{ADDITIONAL INFORMATION}

Correspondence and requests for materials should be addressed to X.W.

Reprints and permission information is available at http://www.nature.com/ reprints

Publisher's note Springer Nature remains neutral with regard to jurisdictional claims in published maps and institutional affiliations.

Open Access This article is licensed under a Creative Commons Attribution 4.0 International License, which permits use, sharing, adaptation, distribution and reproduction in any medium or format, as long as you give appropriate credit to the original author(s) and the source, provide a link to the Creative Commons license, and indicate if changes were made. The images or other third party material in this article are included in the article's Creative Commons license, unless indicated otherwise in a credit line to the material. If material is not included in the article's Creative Commons license and your intended use is not permitted by statutory regulation or exceeds the permitted use, you will need to obtain permission directly from the copyright holder. To view a copy of this license, visit http://creativecommons. org/licenses/by/4.0/.

(c) The Author(s) 2021 